\title{
Automated scoring method for the CDMAM phantom
}

\author{
Yip M. ${ }^{a}$, Chukwu W. ${ }^{a}$, Kottis E. ${ }^{a}$, Lewis E. ${ }^{a}$, Oduko J. ${ }^{b}$, Gundogdu, O. ${ }^{b}$, Young K.C. ${ }^{b}$ and \\ Wells K. ${ }^{a}$ \\ ${ }^{a}$ Centre for Vision, Speech and Signal Processing, Faculty of Engineering and Physical \\ Sciences, University of Surrey, Guildford, Surrey, GU2 7XH, UK; \\ ${ }^{b}$ National Coordinating Centre for the Physics of Mammography, Royal Surrey County \\ Hospital, Guildford, Surrey, GU2 7XX, UK
}

\begin{abstract}
CDMAM phantoms are widely used in the Europe to assess the performance of mammography systems utilising small size and low contrast disc details. However, the assessment of CDMAM images by human observers is slow and tedious. An automated method for scoring CDMAM images (CDCOM) is widely available to address this issue. We have developed an alternative automated scoring tool to score CDMAM images, Quantitative Assessment System (QAS), for similarly removing inter- and intra- observer variability. This provides additional valuable information about the contrast and SNR of each gold disc within the image. The QAS scores CDMAM phantom images using a scanning algorithm. QAS scoring results were compared with human observers and with CDCOM. It was found that QAS was comparable with human observers in scoring, whereas CDCOM consistently scored a higher number of discs correctly in CDMAM images compared with QAS and human observers.

QAS results have been used to analyse the effects of different digital mammography system modulation transfer functions (MTFs) on fine details for a number of systems in the form of contrast degradation factor (CDF) measurements. CDF curves for experimentally acquired CDMAM images were compared with those for simulated CDMAM images to assess the accuracy of contrast measurements.
\end{abstract}

Keywords: Digital Mammography, Simulation, Contrast-detail, CDMAM phantom, Observer Performance

\section{INTRODUCTION}

Performance assessment of mammography systems is a well documented and well understood area, utilising image quality metrics such as MTF (modulation transfer function), NNPS (normalised noise power spectrum) and DQE (Detected Quantum Efficiency). ${ }^{1-3}$ These metrics provide a widely accepted method of characterising the physical performance of detector systems. However, they do not generally include other parts of the imaging chain (e.g. scatter rejection and geometric blurring) or the role of the human observer. The relationship between physical and the psychophysical performance characteristic of the mammographic imaging chain has been investigated in detail but the link to diagnostic performance remains uncertain. ${ }^{3-5}$

Contrast detail measurements provide a means of assessment of the imaging chain that has a dependence on human observer performance. The CDMAM test phantom is used to evaluate contrast detail performance, using a 205 square cell grid with two gold discs within each cell: one in the centre, and one randomly located in one of the four corner quadrants. The discs vary in gold thickness (from $0.03 \mu \mathrm{m}$ to $2.00 \mu \mathrm{m}$ ) and diameter (from $0.06 \mathrm{~mm}$ to $2.00 \mathrm{~mm}$ ) along the rows and columns, respectively. In human reading, the task of the observer is to correctly identify the location of the eccentric disc within each cell. This is a time-consuming task, and has inter- and intra-observer variability in scoring. Therefore, an alternative assessment approach is to use an automated scoring tool known as CDCOM. ${ }^{6}$ However, the exact methodology adopted within the CDCOM framework and further information on the Signal-to-Noise Ratio (SNR) and Contrast-Noise Ratio (CNR) of the detected discs is not available to the user. This has motivated the development of an in-house

Further author information: (Send correspondence to M. Yip)

M. Yip : E-mail: m.yip@surrey.ac.uk, Telephone: +44 (0)1483 689856

\footnotetext{
Medical Imaging 2009: Image Perception, Observer Performance, and Technology Assessment, edited by Berkman Sahiner, David J. Manning, Proc. of SPIE Vol. 7263, 72631A

(C) 2009 SPIE · CCC code: 1605-7422/09/\$18 - doi: 10.1117/12.811657
}

Proc. of SPIE Vol. $726372631 \mathrm{~A}-1$ 
automated scoring software tool (Quantitative Assessment System, QAS) to provide additional information on the discs as these are assessed. From this additional information, data such as SNR and contrast properties of the system can be obtained for further analysis. In this work we present details regarding the contrast degradation of the discs due to detector blurring using data obtained from the QAS tool.

\section{METHODOLOGY}

The task of an automated CDMAM scoring system is to locate the discs within each cell of the CDMAM image. This was divided into two major processing steps:

1. A pre-processing stage, for extraction of grid lines and intersections of the CDMAM image, in order to locate each cell;

2. Implementation of a disc detector algorithm within each quadrant

\subsection{Pre-processing}

The CDMAM image may contain bright areas outside the phantom image which need to be removed before processing, therefore the image is cropped. In order to account for variations in the manufacture of the cells within the CDMAM phantom, search templates need to be correctly aligned for each image being assessed. The CDMAM image was binarised using a Canny edge detector to find the edges of the grid. This searches for the local maxima of the gradient in the image, calculated using a derivative of a Gaussian filter. It uses two thresholds, to detect strong and weak edges, and includes weak edges in the output only if they are connected to the strong edges. The image resolution was reduced by a factor of 2 to speed up processing for a Hough transform to be applied, in order to extract the binarised grid lines from the edge image. Coordinates of the intersection of the lines were then calculated as illustrated in Figure 1(a). The intersections were grouped in a vertical fashion to define the four corners of each of the 205 cells, ready for the implementation of the disc detector algorithm.

\subsection{Implementation of a disc detector algorithm}

Search areas were created and applied to each cell within the grid structure using the intersection information found in the pre-processing stage. The corner search areas were located at a pre-defined position from the centre of the cell, assuming the central disc is at this point. The pre-defined position was assumed to be approximately one third of the distance between the central discs of two adjacent cells as illustrated in Figure 1(b). These search areas were cropped by $85 \%$ to ensure that the finite thickness of the grid lines did not interfere with the disc detection process. Five search areas were defined per cell as shown in Figure 2 and the disc detector algorithm was then applied to each.

Search areas were further eroded to twice the diameter of the disc of interest, with an exception for the smallest discs which may appear in the image as only one pixel in diameter. The search area for the smallest discs (less than $0.20 \mathrm{~mm}$ in diameter) were eroded to 5 times the disc diameter. A circular region, the disc template, equal to the known size of the gold disc in the cell was scanned across the search area, using single pixel steps. The average pixel value within this disc template was calculated. Ideally, signal intensity and contrast should be maximal when the disc is correctly located within the search window.

For each of the decision criteria: maximum contrast and signal, along with its similarity in its signal and contrast to the central disc; a vote is cast upon which corner the algorithm believes the eccentric disc to be in. The vote system, based on the above four criteria, makes a decision on the location of the eccentric disc within each cell.

\section{EXPERIMENT}

There are two objectives in our experiment: first, to compare the performance of QAS to current methods of scoring the CDMAM phantom; second, to use QAS to assess the contrast data of individual discs in experimentally acquired images and compare the results with those simulated.

Proc. of SPIE Vol. 7263 72631A-2 


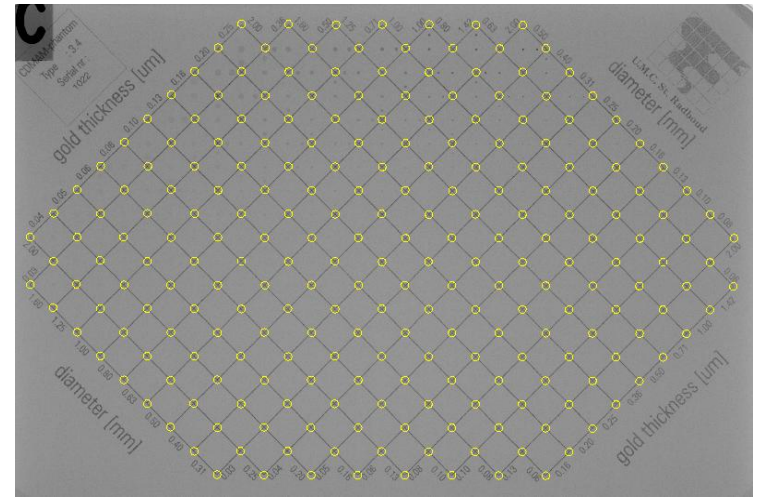

(a)

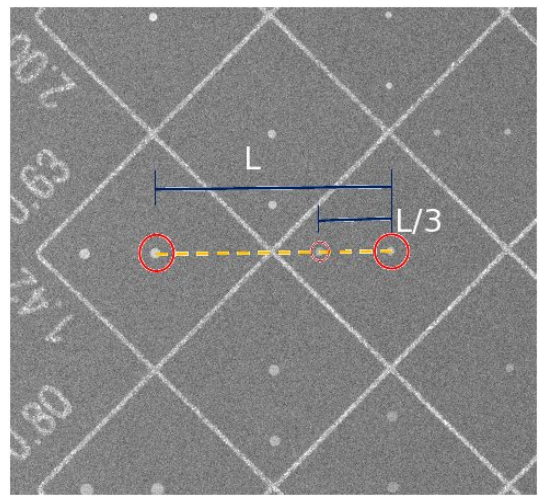

(b)

Figure 1. (a) Illustrates the intersections of the grid within the CDMAM image as found using QAS. (b) Calculation of the corner disc coordinates in relation to the central discs from adjacent cells. The distance between the central disc (large red circle) and the eccentric disc (small red circle) is approximately one third of the distance between the central discs of adjacent cells.
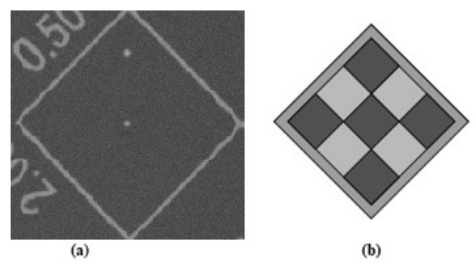

Figure 2. Example of the search area construction (a) Original cell in CDMAM phantom (b) Diagrammatic CDMAM cell: Border shows cell cropped to $85 \%$ of the cell area, to avoid grid lines. Placement of five search areas within the cell is shown with dark squares.

\subsection{CDMAM Simulation}

CDMAM phantom image simulation has been carried out as part of a wider project to simulate digital mammography systems based on image quality metrics. ${ }^{7}$ In brief, the process for image simulation of a CDMAM phantom involved creation of two images: a blurred resampled image and a noise image.

The blurred template image was initially produced using a thresholded version of a CDMAM phantom aforementioned. Pixel values were assigned to the background and foreground details as expected for a specific dose. This idealised image was modified to include the residual heel effect bias before the system's presampled MTF was applied and the image was resampled to the final pixel size. In addition, as the CDMAM phantom image is acquired with the CDMAM phantom at a known distance above the detector face, there is geometric blurring of the image acquired. This was incorporated into the image simulation chain by applying a geometric blurring MTF. ${ }^{7,8}$

A noise image was created through the use of the experimental NNPS data and applied to a Gaussian noise field. To account for non-uniform variance across the image due to structural and geometric variations on photon statistics across the detector face, a variance map was sampled and applied to the noise image. ${ }^{7,9}$ The resulting noise image was is incorporated into the simulated CDMAM phantom image on a pixel-bypixel basis using an empirically measured mean-variance relationship from the system to be simulated. Thus allowing for simulation of images at different doses.

\subsection{Contrast Detail Scoring}

The QAS tool was compared to human observer scoring, as well as the CDCOM scoring for a set of 8 experimentally acquired images for an a-Se system (Hologic Selenia) at one setting (55mAs, 32kVp Molybde- 
num/Rhodium target/filter material). The scoring results were processed as outlined by previous authors ${ }^{10-12}$ to produce contrast-detail curves. Young et $\mathrm{al}^{11,12}$ outlines a method to adjust automated scoring to predict human observer readings. However, for this work, all results were left unmodified, human and automated, thus only 'raw' results were compared for all contrast-detail curves.

The proportion of discs correctly located in each cell was determined from averages from a number of CDMAM phantom images and used to create contrast detail curves. A model fit ${ }^{10}$ was applied to the data, that describes probability of detection $p(d)$ of an object of diameter $d$ as a function of its signal contrast $C=\log \left(1-e^{-\mu d)}\right.$ in the form of a psychometric curve:

$$
p(d)=\frac{0.75}{1+e^{f\left(C-C_{T}\right)}}+0.25
$$

where $f$ is fitted parameter. Curves are fitted to the data using a least squares procedure.

CDCOM and QAS scoring results were further compared for two systems: a CsI phosphor system (GE Essential) and an a-Se system (Hologic Selenia) for a range of doses. Eight images were experimentally acquired for both systems at each dose, keeping the tube voltage the same whilst altering the tube currenttime product.

It is straightforward to estimate the theoretical radiographic contrast of a given thickness of gold in the CDMAM test object configuration for a specific beam quality. However, the contrast measured in images of the discs are also affected by noise, scatter and the detector characteristics, so that there will be discrepancies between radiographic and measured contrasts. In addition, there may be slight variation in the actual thickness of the discs due to manufacturing tolerances, which make it difficult to know the correct expected contrast for each disc. Therefore, we used the MTFs for different systems to incorporate appropriate blurring in idealised (simulated) CDMAM images with discs of known contrasts ${ }^{7}$ to assess the accuracy of contrast measurements. These images were created without noise applied to assess the contrast of the discs more effectively.

\subsection{Extracting Contrast Data}

Contrast data from the correctly located discs in the 8 CDMAM images were collated from the QAS tool and used in the calculation of disc contrast degradation factors ${ }^{8}$ for different digital mammography systems. The contrast degradation factor $(\mathrm{CDF})$ was calculated as a ratio of the contrast of the disc before and after blurring had been applied:

$$
C D F_{M T F}=\frac{C_{\text {blurred }}}{C_{\text {expected }}}
$$

For experimental images, $C_{\text {expected }}$ was calculated theoretically for X-ray spectra with specific beam qualities $(\mathrm{kVp}$, target/filter material) passing through specific gold thicknesses, and the other phantom materials. This did not take account of scatter or noise within the image, and an expected discrepancy in contrast of up to $10 \%$ was expected and verified with measurements from the experimental CDMAM images for large discs. For simulated images, $C_{\text {expected }}$ was used as an input with a contrast-thickness relationship ${ }^{7}$ and the contrast measured again after blurring with the MTF was applied. QAS measures contrast from each disc using the disc template described in section 2.2. Four further ROIs were extracted from the surrounding area to measure the local background pixel value. The contrast was then calculated using:

$$
C=\frac{P_{\text {background }}-P_{\text {disc }}}{P_{\text {background }}}
$$

It would be expected that the contrast measured for differing disc diameters would deteriorate more rapidly for a system with poorer MTF, than that with 'improved' MTF properties. We have currently characterised two types of detectors: amorphous selenium (Hologic Selenia) and CsI phosphor (GE Essential). However, we can apply more general MTF curves to investigate this further. Creating theoretical MTF curves as in 


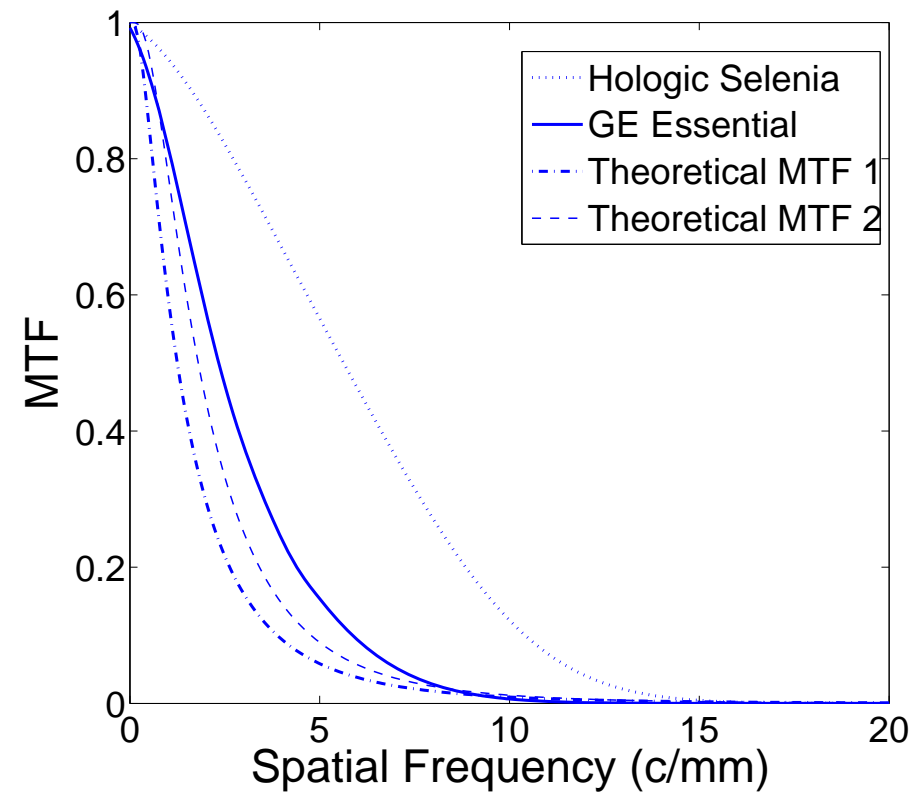

Figure 3. Two theoretical MTF curves derived from the Burgess model alongside experimentally acquired MTF for the GE Essential system and Hologic Selenia system

Saunders and Samei ${ }^{13}$ provides us with a range of MTF curves, that approximate empirically measured CR MTF, with which to test this idea. The MTF model is based on that given by Burgess: ${ }^{14}$

$$
\operatorname{MTF}(u)=0.5 \operatorname{erfc}\left[\alpha \ln \left(u / u_{o}\right)\right]
$$

$\operatorname{erfc}$ is the complimentary error function, $u$ is the absolute value of spatial frequency. $u_{o}$ is an adjustable parameter representing the frequency at which the calculated MTF value is 0.5. $\alpha$ is also an adjustable parameter representing the slope of the curve. Two curves were derived with the Burgess model using parameters $\left[\alpha=0.8 u_{o}=1.25\right]$ (MTF 1)and $\left[\alpha=0.9 u_{o}=1.80\right]$ (MTF2) and are illustrated in Figure 3, with reference to the other experimental MTFs used (Hologic and GE Essential). The spatial frequency at MTF value of 0.5 for the empirically measured systems, in comparison, are found in Table 1. All MTFs have been assumed to be isotropic.

\begin{tabular}{cccc}
\hline Imaging System & Pixel Spacing $(\mu \mathrm{m})$ & MTF $(0.5)(\mathrm{c} / \mathrm{mm})$ & Technology \\
\hline Hologic Selenia & 70 & 5.58 & a-Se \\
GE Essential & 100 & 2.34 & CsI + a-Si \\
MTF 1 & 100 & 1.25 & Theoretical \\
MTF 2 & 50 & 1.80 & Theoretical \\
\hline Table 1. Characteristics of the digital mammography systems modelled
\end{tabular}

Eight CDMAM images with slight rotation of up to 2 degrees have been created using the experimentally acquired MTFs applied, without noise, to compare with the experimentally acquired CDMAM images. A further three CDMAM images were simulated using 29kVp Molybdenum/Rhodium target/filter material for all MTF curves, theoretical and experimental, using a small rotation of up to 2 degrees each.

\section{RESULTS}

Figure 4 shows the QAS tool's contrast-detail curves compared with that of a human observer and with CDCOM. QAS appears to provide better scoring than the human observers. The difference between the 


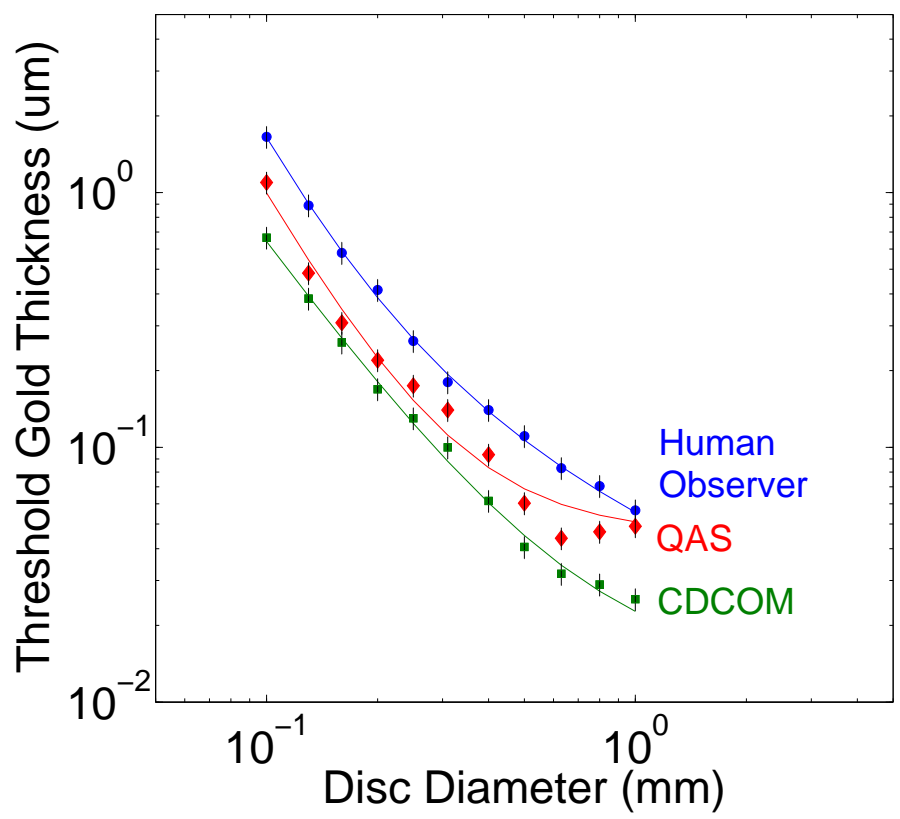

Figure 4. Contrast detail curves for the comparison of QAS with human observers and CDCOM for the Hologic Selenia system. Error bars indicate two standard errors of mean

fitted curves in Figure 4 for QAS and human observers reduces for the larger discs. CDCOM provides consistently higher scoring of discs than QAS. This may be due to CDCOM calculating in a pre-processing step the position of the gold discs in relation to the grid, thus allowing for a much smaller search area. ${ }^{10}$

Table 2 shows the fitted detected threshold gold thickness for all the scoring methods applied to the same set of 8 images. It can be seen that QAS provides a lower threshold of scoring than human observers, whereas the CDCOM can provide scoring up to disc thickness thresholds of approximately a factor of 2 smaller than QAS.

\begin{tabular}{cccc}
\hline Disc Diameter $(\mathrm{mm})$ & \multicolumn{3}{c}{ Threshold Gold Thickness $(\mu m)$} \\
\hline & Human Observer & CDCOM & QAS \\
\hline 1.00 & 0.056 & 0.023 & 0.051 \\
0.80 & 0.067 & 0.027 & 0.054 \\
0.63 & 0.084 & 0.034 & 0.060 \\
0.50 & 0.107 & 0.045 & 0.069 \\
0.40 & 0.138 & 0.061 & 0.083 \\
0.31 & 0.193 & 0.088 & 0.112 \\
0.25 & 0.266 & 0.124 & 0.153 \\
0.20 & 0.387 & 0.181 & 0.225 \\
0.16 & 0.588 & 0.269 & 0.348 \\
0.13 & 0.905 & 0.392 & 0.544 \\
0.10 & 1.645 & 0.642 & 0.996
\end{tabular}

Table 2. Comparison of fitted detection thresholds for differing scoring methods. All scoring is based on the same set of experimentally acquired images from the Hologic Selenia system at 55mAs,32kVp Molybdenum/Rhodium target/filter material.

Figure 5 compares the scoring of the Hologic Selenia system for 3 different settings $(22 \mathrm{mAs}, 55 \mathrm{mAs}$ and $110 \mathrm{mAs}$ at $32 \mathrm{kVp}$ Molybdenum/Rhodium) using QAS and CDCOM. Figure 6 compares the scoring of the GE Essential system for 4 different settings $(14 \mathrm{mAs}, 28 \mathrm{mAs}, 52 \mathrm{mAs}$ and $110 \mathrm{mAs}$ at $29 \mathrm{kVp}$ Molybde- 
num/Rhodium) using QAS and CDCOM.

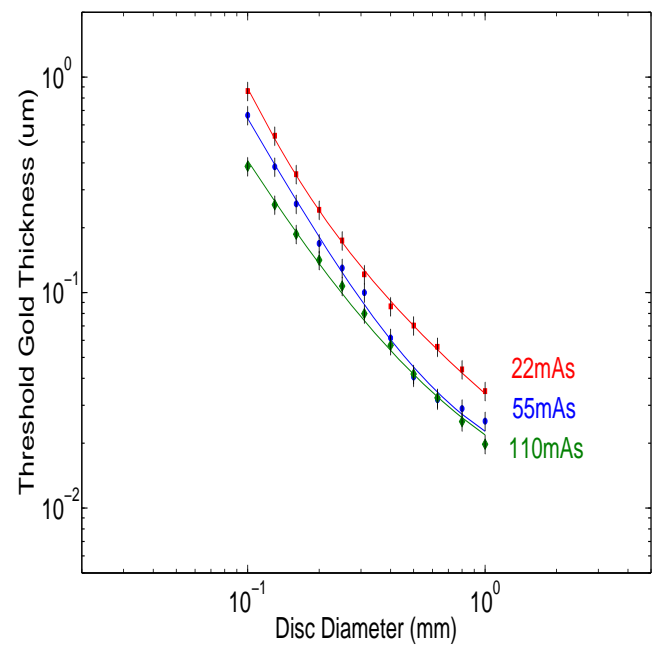

(a)

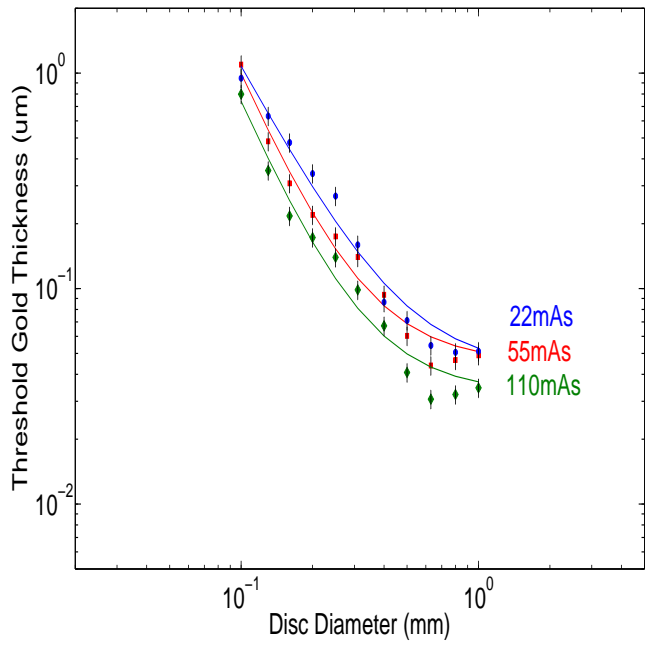

(b)

Figure 5. Contrast detail curves for the Hologic Selenia system using (a) CDCOM (b) QAS. Error bars indicate two standard errors of mean

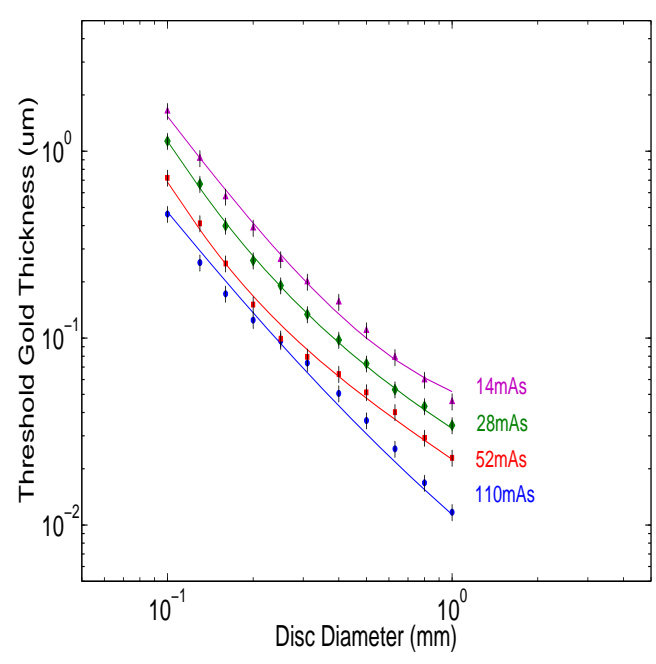

(a)

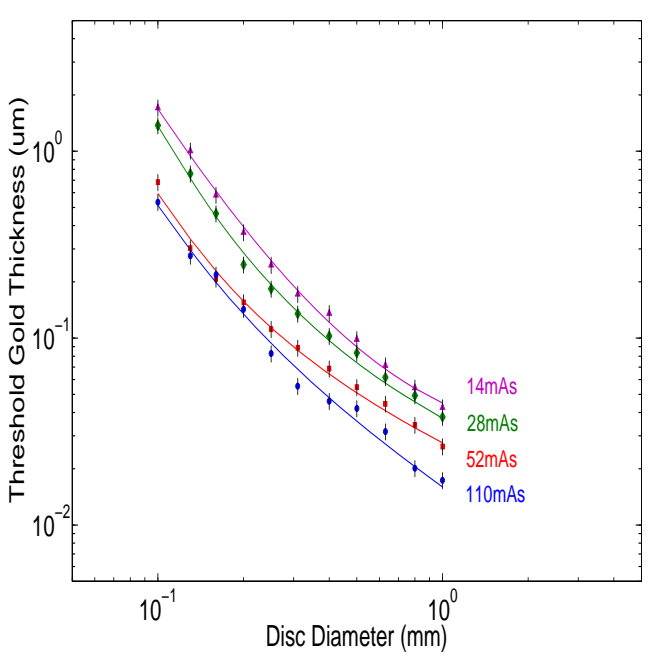

(b)

Figure 6. Contrast detail curves for the GE Essential system using (a) CDCOM (b) QAS tool. Error bars indicate two standard errors of mean

CDF curves for measurement of discs in experimental and simulated CDMAM images are displayed in Figure 7 for two systems. The CDF is approximately equal to unity for large discs and begins to deteriorate rapidly for the smaller discs due to the effect of blurring. Measurements for experimental and simulated images resulted in very similar CDF curves. In both cases, the GE Essential system deteriorates at a larger disc diameter than the Hologic Selenia system, as can be expected since the Hologic Selenia has an improved MTF for all spatial frequencies compared with the GE Essential system (Figure 3). The CDF curve for the Hologic Selenia system begins to deteriorate rapidly, from a CDF value of 0.8 , at approximately $0.31-0.40 \mathrm{~mm}$ for both simulated and experimental data. In comparison, the CDF curve for the GE Essential system begins 
to deteriorate at approximately $0.63 \mathrm{~mm}$. The simulated images resulted in similar CDF data to that obtained from measurements on the experimental images, although there is an average discrepancy of $15 \%$ and $18 \%$ for the Hologic Selenia and GE Essential detectors, respectively. Error bars take account of noise fluctuations in measurement, as well as the errors in the theoretical contrast-thickness relationship. A further source of error may arise from QAS incorrectly locating the exact centre of the disc.

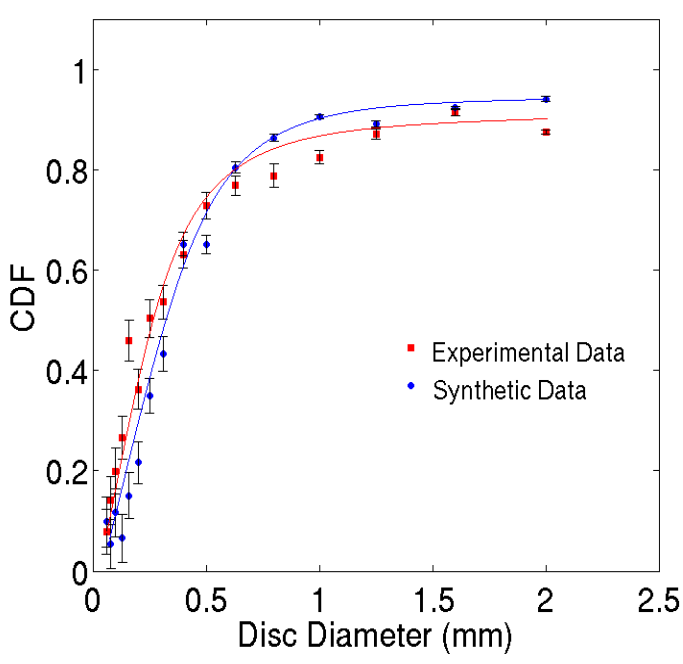

(a)

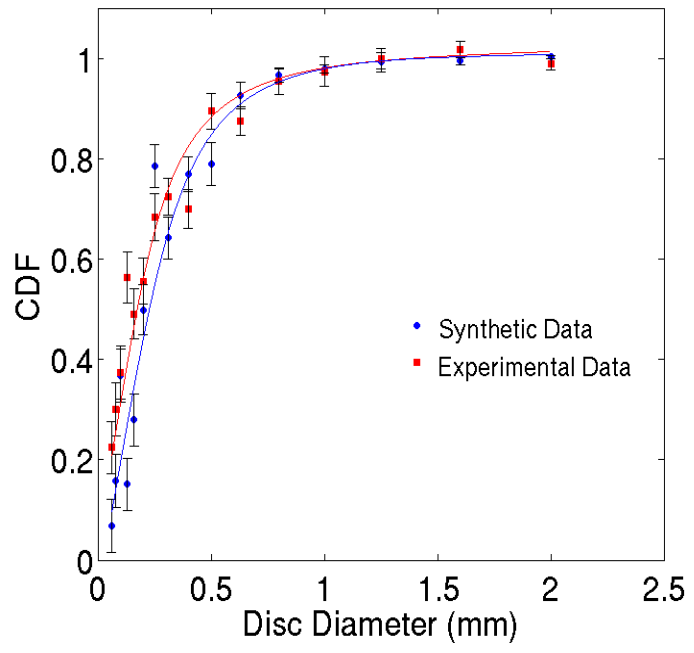

(b)

Figure 7. Comparison of contrast degradation curves for (a) GE Essential system (b) Hologic Selenia system for experimental (red) and simulated (blue) data

In addition to the experimentally acquired contrasts, we compare the contrasts for the MTF curves derived from the Burgess model for comparison. As can be seen in Figure 7, for the systems with poorer MTF, the contrast deteriorates at larger discs than the those with improved MTFs.

Table 3 shows the CDF results displayed in Figure 8. As in Figure 7, the CDF rapidly deteriorates after a CDF value of 0.8. For the Hologic system this occurs at disc diameters between $0.31-0.40 \mathrm{~mm}$. This occurs at a disc diameter of less than $0.63 \mathrm{~mm}$ for the GE Essential, at $0.80 \mathrm{~mm}$ for theoretical MTF 1 , and the CDF for the theoretical MTF 2 rapidly deteriorates from $0.63 \mathrm{~mm}$.

\section{DISCUSSION}

Figure 4 showed that the QAS had a lower threshold of detection when compared with human observer scores for the smaller details. QAS had improved threshold of detection for the larger discs compared with human observers. The QAS was compared with CDCOM on the same images; threshold scoring for the CDCOM was of lower disc thickness compared with QAS in Figure 4. In all cases, the QAS appeared to have difficulty detecting the larger, lower contrast discs. This appears as a lifting in the tail of the contrast detail curves in Figures 4,5 and 6 . CDCOM scores consistently for different doses, evident from the evenly spaced parallel curves in Figures 5(a) and 6(a). The QAS results also display this dose-dependency in its contrast-detail curves. However, there seems to be more variability in its results, which may suggest that QAS requires more images in order to offer reliable readings.

The simulated CDMAM images were created to compare the effect of blurring with known input contrast and measurements were carried out without noise and scatter effects. The contrasts of the discs were obtained from the correctly detected discs and averaged. CDF curves for the experimental and simulated data provided similar results. For the Hologic Selenia system, the simulated data provided a very similar contrast degradation to that measured for the experimental images with an average discrepancy of approximately $15 \%$. 


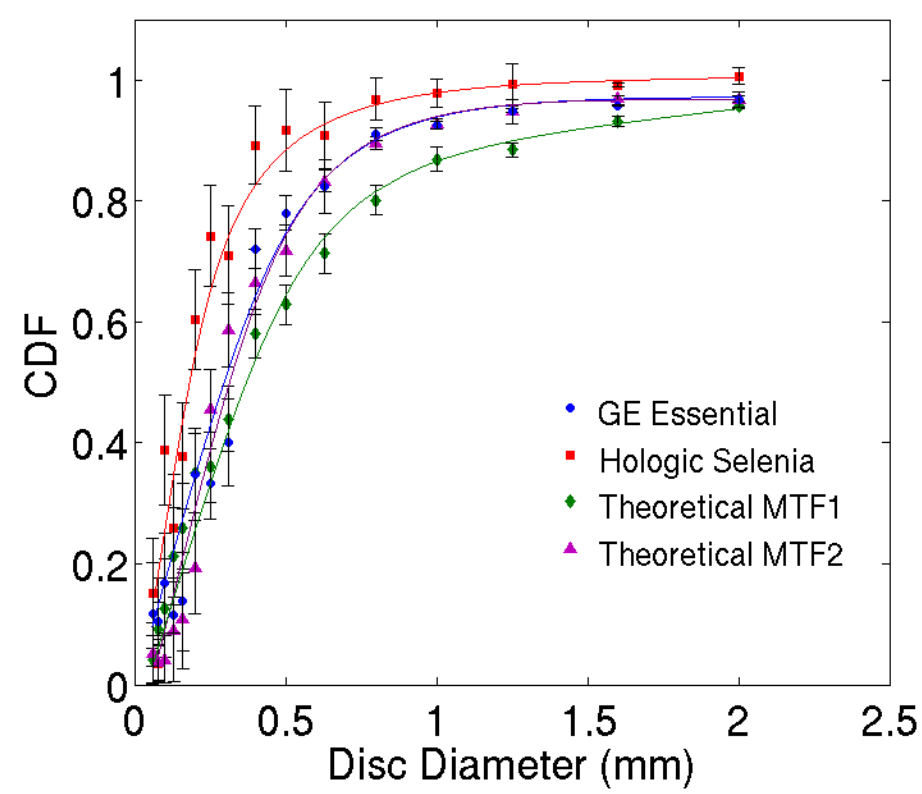

Figure 8. Comparison of contrast degradation curves for all MTF curves displayed in Figure 3. Error bars indicate the standard error of mean. Best fit curves are shown.

\begin{tabular}{ccccc}
\hline Disc Diameter $(\mathrm{mm})$ & Hologic Selenia & GE Essential & Theoretical MTF 1 & Theoretical MTF 2 \\
\hline 2.00 & 1.01 & 0.967 & 0.957 & 0.968 \\
1.60 & 0.991 & 0.958 & 0.932 & 0.970 \\
1.25 & 0.993 & 0.948 & 0.885 & 0.950 \\
1.00 & 0.978 & 0.924 & 0.869 & 0.929 \\
0.80 & 0.969 & 0.911 & 0.802 & 0.896 \\
0.63 & 0.908 & 0.823 & 0.713 & 0.833 \\
0.50 & 0.917 & 0.780 & 0.628 & 0.718 \\
0.40 & 0.892 & 0.721 & 0.581 & 0.666 \\
0.31 & 0.710 & 0.401 & 0.440 & 0.588 \\
0.25 & 0.742 & 0.332 & 0.360 & 0.456 \\
0.20 & 0.604 & 0.348 & 0.350 & 0.194 \\
0.16 & 0.379 & 0.138 & 0.259 & 0.108 \\
0.13 & 0.259 & 0.115 & 0.213 & 0.0909 \\
0.10 & 0.388 & 0.168 & 0.127 & 0.0422 \\
0.08 & 0.0347 & 0.106 & 0.0915 & 0.0372 \\
0.06 & 0.151 & 0.116 & 0.0417 & 0.0528
\end{tabular}

Table 3. Comparison of contrast degradation factors obtained from the QAS tool. Beam quality for all systems were kept at $29 \mathrm{kVp}$, Molybdenum/Rhodium.

Both fitted curves pass through a CDF of 0.8, at approximately $0.31-0.40 \mathrm{~mm}$ disc diameter for the Hologic Selenia system, whereas for the GE Essential it is at a disc diameter less than 0.63mm. Other discrepancies between measurements on experimental and simulated images may be due to very minor inaccuracies in the manufacturing of the gold discs, changing the expected contrast. In addition, although QAS may have detected the correct location of the disc within the cell, upon closer inspection, there was a slight discrepancy between the expected location of the disc template and the actual location due to the search area available to it. Thus there may be further discrepancies in its measurement of the contrast. For the smaller discs this lead to a lower contrast than expected.

The theoretical MTF curves, both poorer than the experimental MTF curves, lead to the deterioration of 
the CDF curve at much larger diameters, approximately $0.80 \mathrm{~mm}$ disc diameter for theoretical MTF 1 and $0.63 \mathrm{~mm}$ for the theoretical MTF 2. Although the theoretical MTF 2 has a slightly poorer MTF than GE Essential, the finer pixel size has reduced the contrast degradation effect.

This work has developed a new automated CDMAM scoring tool which can contribute data towards signal to noise optimisation of digital mammography systems. QAS provides improved scoring in relation to human observers, with further refinements needed in order to be comparable with CDCOM. The main advantage of QAS is that specific data of the correctly detected discs can be extracted for use in image quality experiments, such as the contrast measurements acquired from the QAS tool. These have shown that CDF measurements are indicative of the detector MTFs, illustrating the physical consequence of such blurring effects.

\section{Acknowledgment}

We would like to thank Hirofumi Uemura from Kyushu Institute of Technology, Japan for his advice during his stay at the CVSSP. This work is part of the OPTIMAM Project and was supported by CR-UK \& EPSRC Cancer Imaging Programme in Surrey, in association with the MRC and the Department of Health (England).

\section{REFERENCES}

[1] Bosmans, H., Carton, A.-K., Rogge, F., Zanca, F., Jacobs, J., Ongeval, C. V., Nijs, K., Steen, A. V., and Marchal, G., "Image quality measurements and metrics in full field digital mammography: an overview," Radiat Prot Dosimetry 117(1-3), 120-130 (2005).

[2] Dobbins, J., [Handbook of Medical Imaging, Volume 1, Physics and Psychophysics: Image Quality Metrics for Digital Systems], SPIE Press (2000).

[3] Marshall, N., "A comparison between objective and subjective image quality measurements for a full field digital mammography system," Phys. Med. Biol. 51, 2441-2463 (2006).

[4] Roehrig, H., Krupinski, E., and Yu, T., "Physical and psychophysical evaluation of digital systems for mammography," in [SPIE Medical Imaging], 124-134 (1995).

[5] Beutel, J., Kundel, H., and Bellingham, V., eds., [Handbook of Medical Imaging, Volume 1: Physics and Psychophysics], SPIE Press (2000).

[6] Visser, R. and Karssemeijer, N., Manual CDCOM version 1.5: software for automated readout of CDMAM 3.4 images (2007).

[7] Yip, M., Lewis, E., Young, K., and Wells, K., "Validation of a digital mammography image simulation chain with automated scoring of CDMAM images," in [International Workshop on Digital Mammography, LCNS 5116], 409-416 (2008).

[8] Sandborg, M., Dance, D., and Carlsson, G. A., "Calculation of contrast and signal-to-noise degradation factors for digital detectors in chest and breast imaging," tech. rep., Department of Radiation Physics, Linkoping University (2003).

[9] Marshall, N., "Retrospective analysis of detector fault for a full field digital mammography system," Phys. Med. Biol. 51, 5655-5673 (2006).

[10] Karssemeijer, N. and Thijssen, M., "Determination of contrast-detail curves of mammography systems by automated analysis," in [Digital Mammography], Doi, K., Giger, M., Nishikawa, R., and Scmidt, R., eds., 155-160, Elsevier (1996).

[11] Young, K., Cook, J., Oduko, J., and Bosmans, H., "Comparison of software and human observers in reading images of the CDMAM test object to assess digital mammography systems," in [Proc. SPIE Medical Imaging], Flynn, M. J. and Hsieh, J., eds., 6142(1), 614-206, SPIE (2006).

[12] Young, K., Cook, J., and Oduko, J., "Automated and human determination of threshold contrast for digital mammography systems," in [International Workshop on Digital Mammography LNCS], Astley, S.M., B. M. R. C. Z. R., ed., 4046, 266-272, Springer, Heidelberg (2006).

[13] Saunders, R. and Samei, E., "A method for modifying the image quality parameters of digital radiographic images," Med. Phys. 30(11), 3006-3017 (2003).

[14] Burgess, A. E., "An empirical equation for screen MTFs," Medical Physics 5(3), 199-204 (1978). 